Phase 3: May 2011-September 2011:

A Training package was introduced/spread and Ward Posters and Handover sheet were developed.

Phase 4: October 2011-August 2012:

Monthly run charts of results were shared with senior managers. Pink order slips and orange leaflets were introduced.

Results We achieved our target for 2010/11. The 1.95\% target for $2011 / 2012$ was more difficult but was achieved as shown in the table.

Conclusions In achieving our targets we improved communication and changed the culture from staff not unduly concerned with missed doses to staff taking action to reduce missed doses and improve patient care.

Abstract GRP-157 Table 1

\begin{tabular}{ll}
\hline Date & \% Missed Doses (Target 1.95\%) \\
\hline Nov 2011 & $2.37 \%$ \\
Jan 2012 & $1.88 \%$ \\
Feb 2012 & $1.47 \%$ \\
Mar 2012 & $1.05 \%$ \\
\hline
\end{tabular}

No conflict of interest.

\section{GRP-158 REPORTING AND ANALYSIS OF ERRORS IN CANCER TREATMENT IN THE ANTIBLASTIC DRUGS LABORATORY OF THE EUROPEAN INSTITUTE OF ONCOLOGY}

doi:10.1136/ejhpharm-2013-000276.158

M Milani, V Sirna, I Cò, E Omodeo Salè. European Institute of Oncology, Pharmacy, Milan, Italy

Background The lack of management software for patients undergoing chemotherapy suggested to us that we should investigate errors that have occurred at all stages of the process: prescription, transcription, preparation, distribution and administration of treatment.

Purpose To encourage reports and classify the errors, in order to develop a computerised system of internal management of chemotherapy which can reduce the risk of error at all stages.

Materials and Methods Two reporting channels were established: one for major errors, such as prescriptions or preparations containing incorrect drugs or dosages, improper units of measurement, diluents incompatible with the active ingredient, improper administration. These errors are shared in corporate software with the Risk Management Office.

The second concerns minor errors, prescriptions containing compilation errors, incomplete compilation of the treatment regimen, incomplete administration of treatment; these errors are reported in an internal Excel file.

Results From January to September 2012, 73 major errors were reported from a total of 30406 preparations. Some of these were: prescription of paclitaxel instead of docetaxel, vinorelbine written as vinblastine; preparation of a 5-fluorouracil weekly dose in a two-day infusor, administration of paclitaxel bag to the wrong patient. In $85 \%$ of these cases the intervention of pharmacist avoided the error. 468 minor errors were reported, including 447 prescription errors, 3 transcription errors, 8 for lack of a cheque of the output treatment and 10 for incomplete delivery of the treatment.

Conclusions This analysis allowed us to draw a picture of the most frequent types of error. Most of them concerned the prescription stage, which we hope to minimise with the implementation of a computerised prescribing system. This will also cut down the transcription and administration errors by reading the barcode of the preparation with a patient wristband. The reduced number of preparation errors can be attributed to the use of an automated system for chemotherapy preparation.

No conflict of interest.

\section{GRP-159 RETROSPECTIVE ANALYSIS OF MOST FREOUENT RISK ERRORS RELATED TO INFORMATIZATION SYSTEM FOR PRESCRIBING AND ADMINISTERING MEDICATION}

doi:10.1136/ejhpharm-2013-000276.159

'N Bensalah, ${ }^{2} \mathrm{G}$ Jabaud-Gazin, 'M Badin, 'M Camus. ' $\mathrm{CH}$ Marc Jacquet, Department of medical informatization, Melun, France, ${ }^{2} \mathrm{CH}$ Marc Jacquet, Pharmacy, Melun, France

Background Although implementing an electronic system shows significant functional effects associated with saving time, reducing costs and contributes to a safe medication process by improving patient safety and quality of service, it can also cause confused actions leading to new types of medication errors (MEs).

Purpose To identify and classify the most frequently observed MEs generated by the computerised tool when prescribing (physician order) and administering drugs (nurses' work).

Materials and Methods In June 2011, Orbis Medical (AgfaHealthcare) software was introduced in our hospital for the medication process including integrated electronic prescription, pharmaceutical analysis and administration (4 clinical units representing 107 beds). Different risks of error were identified during pharmaceutical interventions (PIs) recorded between June 2011 and October 2012 and classified according to the French Society of Clinical Pharmacy recommendations. The focus is on MEs related to computerisation.

Results 605 PIs were made on 3933 prescriptions supplied over 466 days. Among these notifications, $1 / 3$ were attributable to the use of the electronic system. Most MEs reported were due to: 1-regarding the prescription: incorrect dose regimen due to selecting the wrong units, incorrect schedule for dose administration, misuse of the commentary zone (free full text related to specific information), redundancy of 2 lines of the same prescribed drug, false interpretation of alert message; 2-regarding administration: failure to record administration, wrong drugs selected to be administered, misuse of the philtre function, single validation for different schedules.

It was noticed that MEs decreased after the staff had used the software for a period of time.

Conclusions Introducing an electronic tool may have an impact on professional practise. Although making healthcare processes safer, it generates new types of iatrogenic harm (other studies have revealed $5-35 \%$ MEs were attributable to computerisation). The introduction of new technology should be accompanied by regular training and evaluation to prevent misuse and potential adverse events.

No conflict of interest.

\section{GRP-160 REUSE OF STERILE IV LIDOCAINE 2\% VIALS IN BERGMANN'S INFUSION IN AN ORTHOPAEDIC DEPARTMENT IN STIP CLINICAL HOSPITAL}

doi:10.1136/ejhpharm-2013-000276.160

'B Lazarova, 'L Mihailova, ${ }^{2} \mathrm{M}$ Lazarova, ${ }^{3} \mathrm{~L}$ Lozanovska Pancevska. 'Clinical Hospital, Hospital Pharmacy, Stip, FYROM; '2Pliva DOOEL, Drug Department, Skopje, FYROM, ${ }^{3}$ Worwgan Pharm, Drug Department, Skopje, FYROM

Background Single-use vials should be used clinically only for one dose for one patient and then discarded or reused under strictly controlled conditions. Certain conditions may justify repacking of single-use vials into smaller doses each intended for a single patient. This process must be performed under aseptic conditions by properly trained operators. 
Purpose To demonstrate the necessity for aseptic technique and conditions and preparation by the pharmacy.

Materials and Methods For a period of six months 15 patients were prescribed Bergman's solution $500 \mathrm{ml}$ to which was added $5 \mathrm{ml}$ pentoxiphylin and $12 \mathrm{ml}$ lidocaine $2 \%$ (50 ml vials divided between 4 patients) in the orthopaedic department. This infusion was prepared in the nursing room, by the nurses without suitable aseptic conditions. For the next six months pharmacists prepared this infusion in the hospital pharmacy aseptic facility. 17 patients in the orthopaedic department got this solution.

Results The nurses used each $50 \mathrm{ml}$ vial of lidocaine for several patients until the vial was used. The vial was saved for use the following day after initial entry. Within days of application 8 patients required antibiotics and prolonged hospitalisation. Microbiological tests showed MRSA infection. One of the nurses forgot to wash hands before preparing the infusion for 3 patients, one used the same needle for both drugs for 4 patients, and one accidentally touched the needle in 1 patient. In the next six months the hospital pharmacy prepared 17 infusions for 17 patients in the aseptic facility. All patients finished their treatment in very good condition without any complications.

Conclusions Nurses' rooms and training are unsuitable for reusing single dose vials for several patients. Subdividing must follow highly controlled environmental conditions, with training and qualifications of personnel and procedures for reuse, which are met by the hospital pharmacy and pharmacists in our hospital.

No conflict of interest.

\section{GRP-161 RISK ASSESSMENT FORMS FOR PHARMACY PREPARATION}

doi:10.1136/ejhpharm-2013-000276.161

Y Bouwman. Royal Dutch Pharmacists Association, Scientific Institute for Dutch Pharmacists, Den Haag, The Netherlands

Background Pharmacists are allowed to prepare medicines for the needs of patients. They have to balance the benefits and risks of the clinical and pharmaceutical qualities. In other words they have to perform a risk assessment for extemporaneous preparation as well as for stock preparation.

To perform a risk assessment the pharmacist should be able to list the benefits and risks and needs a tool to balance them. Some approaches have been published, but they don't deal with all aspects in one view. We think there is a need for a risk assessment tool that is simple, transparent and conclusive and that deals with all relevant aspects.

Purpose To analyse the pharmaceutical process for decisive steps, levels of evidence and actors. To incorporate these aspects into a practicable form.

Materials and Methods 15 years of feedback from community and hospital pharmacists on former assessment forms, discussions with authorities, 40 years searching for sound reasons for pharmacy preparation, writing an opinion on the Resolution on pharmacy preparation of the Council of Europe, have been used as an input for creating a new form that emphasises the benefit and risk balance.

Results Two forms were developed for the pharmacist: for extemporaneous and for stock preparation. They use the same type of benefit and risk aspects but extemporaneous preparation affects an assignable patient and the request is from an assignable physician. Often two pharmacists are involved, the attending pharmacist and the preparatory pharmacist. All four carry responsibility but the preparatory pharmacist has to decide whether to fulfil the request or not. For stock preparations the preparatory pharmacist will put together the information about benefits and risks. The physician, patient and attending pharmacist have to balance them. Stock preparation requires numerous items per batch and serves a number of patients. This requires a higher level of evidence about the clinical value and a higher quality of design.

Conclusions Forms were developed for the risk assessment of extemporaneous and stock preparations. They show decisions and provide transparency, pointing at responsibility and accountability. Practical experience will provide more information about the roles of pharmacist(s), physician and patient.

No conflict of interest.

\section{GRP-162 RISK CATEGORIZATION OF STANDARDISED CONTINUOUS INJECTION/-INFUSION SOLUTIONS AT THE UNIVERSITY MEDICAL CENTER MAINZ}

doi:10.1136/ejhpharm-2013-000276.162

B Zeiter, S Bellers, I Kraemer. University Medical Center, Pharmacy Department, Mainz, Germany

Background The prescription, preparation and use of parenteral solutions are complex processes composed of many steps, during which mistakes can occur. However, by means of the National Patient Safety Alert 20 (NPSA 20), a risk evaluation of continuous injection/-infusion solutions can be performed.

Purpose To evaluate the risks associated with the intravenous drug treatment of intensive care unit patients at the University Medical Center Mainz. We planned to use the results to identify high-risk products and implement measures to reduce potential risks.

Materials and Methods The NPSA 20 defines eight different risk factors for the evaluation of overall risk. The risk evaluation was conducted for 78 continuous injection/-infusion solutions used in intensive care unit patients. These parenteral solutions are used in standardised concentrations; 16 of them were prepared as ready-touse products in the hospital pharmacy. The potential risks of these 16 preparations were compared with the risks of those not prepared centrally in the hospital pharmacy department.

Results The risk evaluation of the 78 continuous injection/infusion solutions revealed that most of the standardised 78 solutions were moderate-risk products $(68 \%)$. Other solutions were classified as low-risk products (26\%). Only $6 \%$ of the solutions were high-risk products. The favourable results of the risk analysis can be explained by the hospital-wide use of standardised concentrations. Doses are adjusted by using the infusion rate. For a number of products $(12 \%)$ the risk category was downgraded from moderate to low, since ready-to-use products were prepared in the hospital pharmacy department.

Conclusions Out of 78 drug products administered as continuous injection/-infusion solutions to intensive care unit patients only $6 \%$ were categorised as high-risk. This favourable result is explained the use of standardised concentrations and preparation of ready-to-use products in the pharmacy department.

No conflict of interest.

\section{GRP-163 RISK MANAGEMENT MEASURES TO PREVENT PHYSICAL- CHEMICAL INCOMPATIBILITIES DURING CONTINUOUS IV INFUSION}

doi:10.1136/ejhpharm-2013-000276.163

${ }^{1} \mathrm{C}$ Linguadoca, 'S Parazza, 'E Musi, 'A Zanardi, ' $\mathrm{F}$ Caliumi, ${ }^{2} \mathrm{MV}$ Antonini, ${ }^{2} \mathrm{ML}$ Caspani. ${ }^{1}$ Azienda Ospedaliero-Universitaria Parma, Servizio di Farmacia e Governo Clinico del Farmaco, Parma, Italy; ${ }^{2}$ Azienda Ospedaliero-Universitaria Parma, I Servizio di Anestesia e Rianimazione, Parma, Italy

Background Patients in critical care (ICU) settings usually require multiple medicines administered as continuous IV infusions. As a reliable IV access is often unavailable, simultaneous administration through the same line is performed using a Y-site connector 